\title{
Im Schatten der Krise: Über Normativität in der politikwissenschaftlichen Hochschullehre
}

\author{
Mischa Hansel · Daniel Lambach • Julia Reuschenbach
}

Online publiziert: 6. November 2018

(C) Deutsche Vereinigung für Politikwissenschaft 2018

Seit geraumer Zeit ist im Zuge neuer populistischer Bewegungen, dem Erstarken autoritärer Regime sowie dem wahrgenommenen Scheitern liberaler „Grand Strategies“ (Freihandel, Demokratisierung) viel von einer Krise des Liberalismus und liberaler Ordnungsmodelle zu hören. Die Politikwissenschaft, die in der Bundesrepublik einmal als Demokratiewissenschaft gestartet war, muss sich dabei ohne Frage besonders angesprochen fühlen, wie unlängst auch Bundespräsident Steinmeier auf dem DVPW-Kongress „Grenzen der Demokratie“ betont hat (Bundespräsidialamt 2018). Heute ist zwar der direkte demokratische Erziehungsauftrag zurückgetreten. Doch mit dem Selbstverständnis einer Orientierungswissenschaft (vgl. Waschkuhn 2002) verbindet sich ein nicht minder großes Versprechen: Dem gefühlten Verlust von intersubjektivem Sinn etwas entgegensetzen zu können, auf das sich politisch mündige Bürgerinnen und Bürger einigen könnten. Strittig ist allerdings, welche Relevanz die Disziplin dabei im öffentlichen Raum überhaupt hat (Masala 2017). Ebenso ließe sich aber auch argumentieren, dass der eigentliche Orientierungsbeitrag der Politikwissenschaft in ihrem universitären „Alltagsgeschäft“, also in Seminaren, Übungen und Vorlesungen, stattfindet und dort zukünftige Aktivisten, Medienschaffende und politische Verantwortliche adressiert (Eriksson und Sundelius 2005).

Können oder müssen sich Politikwissenschaftlerinnen und Politikwissenschaftler im Seminarraum deutlicher positionieren? Worin bestünde diese Positionierung? Etwa nur in der Erklärung oder auch in der Bewertung der (oder mehrerer) Krise(n)?

\footnotetext{
M. Hansel $(\bowtie)$

Aachen, Deutschland

E-Mail: mischa.hansel@ipw.rwth-aachen.de

D. Lambach

Frankfurt am Main, Deutschland

J. Reuschenbach

Bonn, Deutschland
} 
Anders gefragt: Müssen Politikwissenschaftlerinnen und Politikwissenschaftler auch ,anecken“ und bestimmte Normen und Werte - ungeachtet einer immer heterogeneren Studierendenschaft und des Kontroversitätsgebotes ${ }^{1}$ - als nicht verhandelbar erklären? Ist politikwissenschaftliche Lehre notwendigerweise und immer normativ? Worin manifestiert sich diese Normativität - in der Auswahl der Themen sowie Autorinnen und Autoren, in der Methodik, im Habitus der Studierenden und Dozierenden? Diese wenigen Fragen suggerieren schon, wie schwierig es ist, Normativität in der politikwissenschaftlichen Lehre auf den Punkt zu bringen. Zugleich wird schnell klar, dass solche Fragen am Selbstverständnis der Disziplin rühren. Wer im politikwissenschaftlichen Studium primär eine analytisch-methodische Ausbildung sieht, wird die Frage sicherlich anders beantworten, als jemand der den Schwerpunkt eher auf den Erwerb von Demokratiekompetenz legt.

Auffällig ist jedoch, dass dieser Dissens und überhaupt unsere Vorstellungen einer mehr oder weniger normativen Lehre eher selten thematisiert werden. Das liegt wohl nicht allein an der fortwährenden Reputationsasymmetrie zwischen Forschung und Lehre. Vielmehr sind Dozierende einem Spannungsfeld ganz unterschiedlicher Ansprüche, Leitbilder und Kritiken ausgesetzt, die nicht leicht miteinander zu vereinbaren sind. Da ist zum einen der legitime Wunsch, unterschiedliche Lebensrealitäten der heutigen Studierenden auch dadurch anzuerkennen, dass in der Lehre verschiedene prä-theoretische Weltsichten wertgeschätzt werden, wie Dannica Fleuß in ihrem Beitrag ,Politische Theorie anwendungsbezogen lehren - Lehrende zwischen normativer Zurückhaltung und kritischer Stellungnahme“ argumentiert. In dieser Grundhaltung steckt selbst ein normatives Postulat, das auf das Zurückhalten von Werturteilen seitens der Dozierenden hinausläuft. Zugleich wird die Frage aufgeworfen, worin dann ein minimaler Grundkonsens bestehe und wie mit extremen Positionen umgegangen werden solle. Zweitens ist der Umstand zu erwähnen, dass interpretative Methoden und sprachlich-diskursive Forschungsgegenstände in der Politikwissenschaft stark an Bedeutung gewonnen haben. In der Folge ist die Vorstellung autoritativer Wissensbestände sowie wissenschaftlicher „Fakten“ problematisch geworden (Daase und Deitelhoff 2017). Es wäre verwunderlich, wenn sich dieser Relativismus nicht auch in der Lehre niederschlagen würde und dann - neben Theorien und Analysemethoden - nicht auch die Vorstellung ,gesicherter“ Soll-Aussagen problematisch erscheinen lassen würde. Drittens passt das Rollenmodell der Dozierenden, die eben auch zu lernende Soll-Aussagen definieren, nicht zu den Kernaussagen konstruktivistischer Lehr-Lerntheorien und zur Priorisierung von Kompetenzerwerb und selbstbestimmtem Lernen. Zumindest bedeutet der damit einhergehende Paradigmenwechsel, die Bereitschaft, Kontroll- und Interventionsmöglichkeiten - auch bezüglich normativer Lehrinhalte - abzugeben, womit sich der Beitrag von Julian Eckl ,Theorien als latente Quellen von Normativität und Verantwortung in der Hochschullehre: Die Bedeutung von didaktischen und fach-

\footnotetext{
1 Das Kontroversitätsgebot geht auf den Beutelsbacher Konsens von 1976 zurück, der bis heute gültige Grundsätze der politischen Bildung formulierte. Demnach sollen in Politik und Wissenschaft strittige Inhalte auch im Politikunterricht (sowie in der politikwissenschaftlichen Lehre) kontrovers dargestellt und diskutiert werden.
} 
spezifischen Theoriedebatten am Beispiel des politikwissenschaftlichen Teilgebiets ,Internationale Beziehungen“" unter anderem beschäftigt.

Viertens werden (nicht nur) Politikwissenschaftlerinnen und Politikwissenschaftler im Rahmen populistischer Anti-Eliten-Diskurse „,mitverhaftet“ und es wird ihnen eine liberal-kosmopolitische erzieherische Agenda unterstellt. Die Kritik ist also, dass Politikwissenschaftlerinnen und Politikwissenschaftler schon grundsätzlich zu stark normativ argumentieren. Hier liegt der Impuls nahe, sich dann doch auf die vermeintlich analytische, „faktische“ Ebene zurückzuziehen, um sich als „Wissenschaft“" gegen den Vorwurf der Parteilichkeit zu immunisieren. Der vielerorts durchgeführte „March for Science“,2 der Wissenschaft als Faktenwissenschaft gegen die ,alternativen Fakten' Donald Trumps und anderer Populisten behaupten will und ,evidence-based policy-making“ befürwortet, geht in diese Richtung. Dabei ist fraglich, ob die Negation normativer Inhalte sinnvoll und überzeugend ist, dies gilt sowohl im Seminar in der Hochschule als auch in der breiten Öffentlichkeit.

Ein „Zuviel an Normativität“ - wenn auch mit anderer Intention - schlägt Lehrenden schließlich auch von Seiten politisch aktiver Studierender entgegen, die gröBeren Einfluss auf die Auswahl von Themen sowie Autorinnen und Autoren und im Allgemeinen eine stärkere Berücksichtigung alternativer Perspektiven einfordern, etwa postkolonialer oder feministischer Ansätze. Dem ist insofern zuzustimmen, als die Entscheidung darüber, was als wichtiges - oder gar kanonisches - Thema oder welche Perspektive zu gelten habe, in der Tat eine zutiefst normative ist. Dieser Sachverhalt wird nun - viel stärker im angelsächsischen Raum - politisiert und als Herrschaftsmittel kritisiert. Es geht hier vor allem um latent normative Inhalte, die aber möglicherweise umso prägender sind. Ob diese neuen Ansprüche nun zurückgewiesen oder konstruktiv aufgenommen werden, jedwede Reaktion darauf basiert wiederum auf normativen Vorstellungen politikwissenschaftlicher Lehre und den damit einhergehenden Rollenverständnissen.

Was folgt nun aus diesen konträren Normativitätszuschreibungen und aus dem Spannungsbogen zwischen Positionierung und Zurückhaltung? Zunächst einmal wohl ganz grundlegend die Notwendigkeit einer expliziten Reflexion darüber, wie und wodurch politikwissenschaftliche Lehre normativ ist, sein kann und sollte. Das gesammelte Forum zum Thema „Normativität in der Lehre“ im vorliegenden Heft der PVS möchte zu dieser Diskussion wichtige Impulse beisteuern. Die Beiträge stammen aus unterschiedlichen Teilgebieten des Faches, sind sich aber darüber einig, dass politikwissenschaftliche Lehre immer notwendigerweise normativ ist. Sie begreifen es als Kernaufgabe der politikwissenschaftlichen Lehre, nicht nur eine analytische, sondern auch eine normative Urteilskompetenz der Studierenden zu fördern. Diese kann nur mit normativen Argumenten oder der intensiven Analyse normativer Vorstellungen und Prozesse „trainiert werden“. Zugleich setzen die Beiträge unterschiedliche Akzente in Bezug darauf, worin sich die Normativität politikwissenschaftlicher Lehre manifestiert und wie sie didaktisch einzubetten ist. Schließlich fällt überdies auf, wie stark die Geschichte und das Selbstverständnis der Subdisziplinen zum Verständnis von Normativität beigetragen haben. Dennoch sind die angesprochenen Herausforderungen und Problemlösungsvorschläge von so all-

\footnotetext{
2 Siehe https://marchforscience.de/. Zugegriffen: 20. September 2018.
} 
gemeiner Natur, dass sich unschwer Beispiele auch aus anderen Teilgebieten finden ließen. ${ }^{3}$

Dannica Fleuß plädiert in Ihrem Beitrag einerseits für eine anwendungsbezogene Lehre normativer politischer Theorie und andererseits für eine normative Zurückhaltung der Lehrenden. Eingangs differenziert sie zwischen den ,externen Quellen von Normativität in Lehrveranstaltungen", gemeint sind Studienordnungen oder Lehrmethode, sowie den normativen Lerngegenständen, wie beispielsweise Menschenrechtskonzepten. Ihr Fokus liegt auf den Lerngegenständen und deren Vermittlung durch Analyse von konkreten Entscheidungs- und Dilemmasituationen. Hierdurch werde einerseits der Kompetenzerwerb der Studierenden gefördert. Andererseits erfolgt durch die Konkretisierung ein stärkerer Druck seitens der Lehrenden, Werturteile auszusprechen und es besteht die Gefahr, dass der normative Bias der Lehrenden zu stark auf die Lehr-Lernsituation einwirkt und das Prinzip des demokratischen Pluralismus verletzt. Um dem entgegenzuwirken, entwickelt Fleuß ein Konzept des „Case-Teaching“, mithilfe dessen Studierende in die Rolle der oder des normativ Urteilenden versetzt werden.

Im Unterschied zu Dannica Fleuß fokussiert Julian Eckl weniger auf normative Lehrgegenstände und dafür umso mehr auf ,latente Quellen von Normativität““. Dabei gelte es zu erkennen und zu reflektieren, in welchem Maße in didaktischen und fachspezifischen Theoriedebatten normative Aspekte mitverhandelt würden. Theorien der internationalen Beziehungen enthalten schon darum einen normativen Kern, dass sie bestimmte Gegenstände als wichtig oder unwichtig markieren, bestimmte Handlungsweisen als „Normalfall“ charakterisieren und dadurch politisch instrumentalisierbar sind. Verschiedene Lehr-Lern-Theorien wiederum modellieren die ideale Rolle und den Verantwortungsrahmen der Dozierenden ganz unterschiedlich. So wird deutlich, dass konstruktivistische Lerntheorien in viel geringerem Maße Instruktionen und Interventionen der Dozierenden befürworten als behaviouralistische Lerntheorien. Hier geht es zudem nicht mehr um die Vermittlung spezifischer Wissensbestände, sondern um die Schaffung einer Lernumgebung, in der Perspektivwechsel angeregt und das Hinterfragen von Realitätskonstruktionen ermöglicht wird. Dies lässt freilich die Frage entstehen, ob dann auch gesellschaftliche Grundkonsense als eine (unter mehreren möglichen) Realitätskonstruktion(en) abgelehnt werden können, beziehungsweise wie und von wem auf extreme Positionen reagiert werden kann.

Der Beitrag von Eva Ottendörfer, Marika Gereke und Tanja Brühl „Mehr Normativität wagen: Ein Plädoyer für eine reflexive Grundhaltung in der politikwissenschaftlichen Lehre" beruft sich explizit auf die konstruktivistische Lehr-LernTheorie und plädiert entschieden für ein „Mehr an Normativität“ in der politikwissenschaftlichen Hochschullehre, verbunden mit einer reflexiven Grundhaltung und der expliziten Offenlegung normativer Haltungen durch Lehrende und Studieren-

\footnotetext{
3 In der Regierungslehre etwa scheint eine normative Verortung im Umgang mit tagespolitischen Fragen, die den Studierenden auch in ihrer Alltagswelt begegnen (bspw. rechtsradikale Proteste und Ausschreitungen, europaskeptische Kampagnen), beinahe unumgänglich. In der vergleichenden Systemlehre wiederum kann intensiv darüber gestritten werden, inwiefern explizit oder implizit europäisch inspirierte Idealtypen und Vergleichsmaßstäbe legitim sind oder einen problematischen normativen Bias darstellen.
} 
de. Dabei kommt der Friedens- und Konfliktforschung zugute, dass sie sich, anders als die Teildisziplin der Internationalen Beziehungen, von Beginn an als normativkritische Wissenschaft verstanden hat, die insbesondere für Machtfragen auch in der Lehrsituation sensibel ist. Gerade unter diesem Gesichtspunkt wird der Rollenwandel der Lehrenden begrüßt, von einer Autorität der Wissensvermittlung zu einer Rolle als Begleiterin und Begleiter, Beraterin und Berater oder Moderatorin und Moderator. Dennoch impliziert diese Rolle immer noch ein Machtverhältnis, das schon in der Themen- und Methodenwahl begründet ist. Umso wichtiger ist es, die eigenen normativen Haltungen offenzulegen und den Studierenden eigene Reflexionsprozesse zu ermöglichen, etwa indem sie in die Rolle von Expertinnen und Experten schlüpfen.

\section{Literatur}

Bundespräsidialamt. 2018. Rede des Bundespräsidenten auf dem 27. Kongress der deutschen Politologen. http://www.bundespraesident.de/SharedDocs/Reden/DE/Frank-Walter-Steinmeier/Reden/2018/ 09/180926-DPVW-Kongress-Frankfurt.html (Erstellt: 26. September 2018). Zugegriffen: 01. Oktober 2018 .

Daase, Christopher, und Nicole Deitelhoff. 2017. Populismus und Lüge: Politikwissenschaft in Bedrängnis. Zeitschrift für Internationale Beziehungen 24(2). Editorial vom 02.10.2017.

Eriksson, Johan, und Bengt Sundelius. 2005. Molding minds that form policy: how to make research useful. International Studies Perspectives 6(1):51-71.

Masala, Carlo. 2017. Auf dem Rückzug. Zeit Online, 23.02.2017.

Waschkuhn, Arno. 2002. Grundlegung der Politikwissenschaft: Zur Theorie und Praxis einer kritischreflexiven Orientierungswissenschaft. München: Oldenbourg. 